\title{
MEMS-BASED VARIABLE CAPACITOR FOR MILLIMETER-WAVE APPLICATIONS
}

\author{
Zhiping Feng ${ }^{1}$, Huantong Zhang ${ }^{1}$, Wenge Zhang ${ }^{2}$, Bingzhi Su ${ }^{2}$, \\ K. C. Gupta ${ }^{1}$, Victor M. Bright ${ }^{2}$, and Y.C. Lec ${ }^{2}$ \\ NSF Center for Advanced Manufacturing and Packaging of \\ Microwave, Optical, and Digital Electronics (CAMPmode) \\ ${ }^{1}$ Department of Electrical and Computer Engineering \\ ${ }^{2}$ Department of Mechanical Engineering \\ University of Colorado, Boulder, CO 80309-0427 \\ http://mems.colorado.edu
}

\begin{abstract}
We have demonstrated a MEMS-based variable capacitor with outstanding RF performance acceptable for millimeter-wave applications. Actuators were used to move the MEMS capacitor plate vertically to change the air gap between the MEMS plate and the signal line of a coplanar waveguide (CPW). The air gap change resulted in a capacitance change with a ratio of $2.7: 1$. The MEMSbased capacitor achieved a very impressive quality factor $(Q)$ that was close to 300 at $0.1 \mathrm{pF}$ and $10 \mathrm{GHz}$. In addition, the capacitance showed no resonance and the equivalent resistance was less than $0.5 \Omega$ in the frequency range up to $40 \mathrm{GHz}$. The assembly technology and the RF and actuator designs critical to the capacitor demonstration are discussed.

\section{INTRODUCTION}

Microelectromechanical systems (MEMS) allow precise positioning and repositioning of suspended membranes and cantilevers that can be integrated with radio frequency (RF) circuits for tuning and switching. One of the important applications of MEMS in RF circuits and antennas is in the form of tunable capacitors. High Q-factor capacitors are needed in microwave communication systems to replace the scmiconductor varactors. In MEMS, mechanical tuning avoids the high losses associated with semiconductors at high frequencies. Polysilicon as the structure material in MEMS with a gold layer and air instead of other dielectric materials make high-Q capacitors possible. Also, it is possible to design MEMS with linear movement (with respect to the control signal), thus allowing linearly tunable capacitance.
\end{abstract}

Research on the MEMS tunable capacitors is underway [1-4]. Larson et al. designed a MEMS tunable interdigitated capacitor using an electrostatic actuator [1]. The capacitance could be changed from $0.035 \mathrm{pF}$ to $0.1 \mathrm{pF}$ with the bias voltages of $80 \mathrm{~V}$ to $200 \mathrm{~V}$. Dec et al. [2,3] have reported a MEMS tunable parallel plane capacitor using, again, an electrostatic actuator. A Q-factor of 9.6 at $1 \mathrm{GHz}$ for a $4 \mathrm{pF}$ capacitor has been reported. The capacitance value changed from $4.0 \mathrm{pF}$ to $4.4 \mathrm{pF}$ for bias voltage 0 to $0.8 \mathrm{~V}$. Young and Boser [4] have described a MEMS tunable capacitor with electrostatic actuators using aluminum as the structural material and obtained a $Q$ value of 62 at $1 \mathrm{GHz}$ for a capacitance of $2.11 \mathrm{pF}$ (4 shunt capacitors in parallel) up to 1.2 $\mathrm{GHz}$. However, all these MEMS devices were developed for microwave applications. It is equally important to develop MEMS variable capacitors for millimeter-wave applications.
In this study, we have demonstrated a MEMS-based variable capacitor with outstanding RF performance acceptable for millimeter-wave applications. The capacitor is shown in Figure 1. More importantly, we have developed the variable capacitor using. existing silicon-MEMS foundry MUMPS (Multiple User MEMS Processes). The MEMS structure on the lossy host silicon substrate was transferred to a low-loss ceramics substrate. Such a flip-chip assembly and transfer process was the key technology to assure the excellent millimeter-wave performance of our devices. In addition, the RF and the actuator designs were critical considerations. The assembly technology and the MEMS designs are described in the following sections.
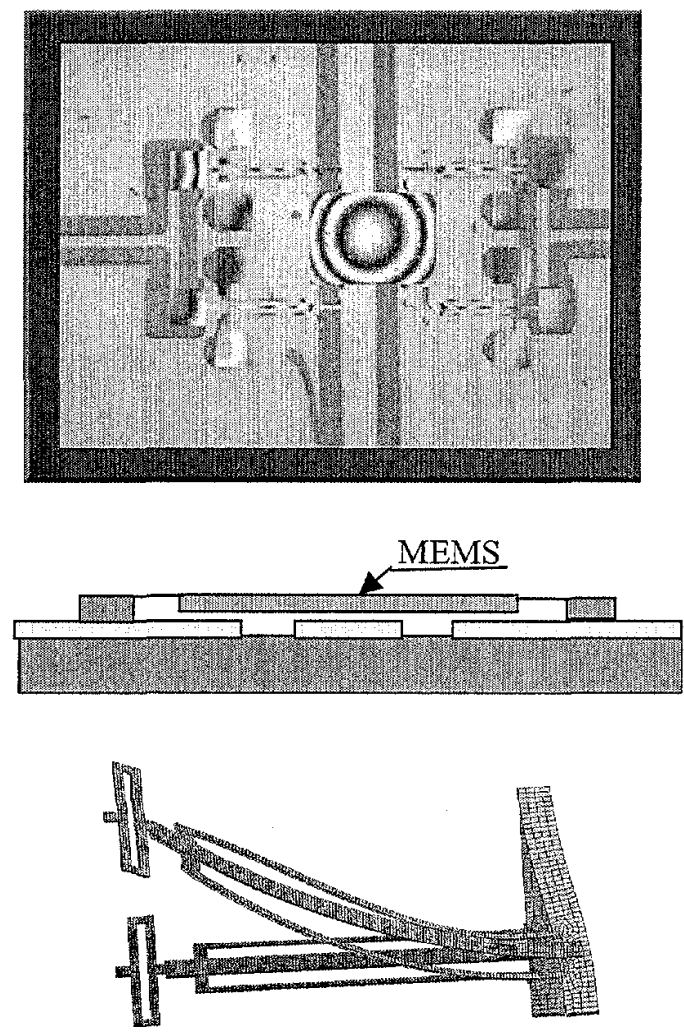

Figure 1: MEMS-based variable capacitor driven by vertical electro-thermal actuators. 


\section{ASSEMBLY}

Figure 1 shows the capacitor driven by four vertical electrothermal actuators. The actuators move the MEMS plate vertically to change the air gap between the MEMS and the signal line of a coplanar-waveguide (CPW). For good manufacturability, we decided to use foundry-fabricated MEMS. Unfortunately, a typical MEMS foundry process, e.g. MUMPS for this study, uses lowresistivity silicon substrates that are lossy at high frequencies. As a result, a new assembly technology has been developed to transfer the MEMS from the host silicon substrate to a new ceramics substrate. Referring to Figure 2, after flip-chip bonding, hydrofluoric acid (HF) is used to removed $\mathrm{SiO}_{2}$ sacrificial layers that are placed between the MEMS and the host silicon substrate. The process was reported in detail in $[5,6]$. After transferring, the MEMS device made of polysilicon and gold layers can perform well up to $40 \mathrm{GHz}$ if the actuator and the RF designs are appropriately considered.
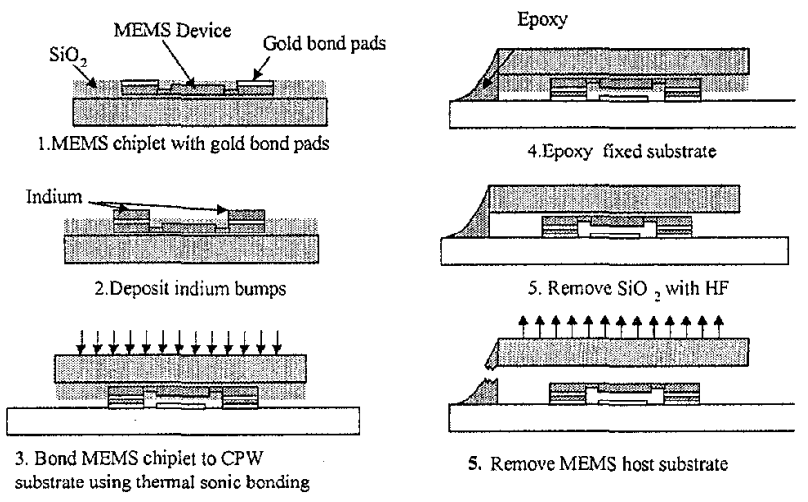

5. Remove MEMS host substrate

Figure 2: Flip-chip assembly and silicon removal for RF MEMS

\section{ACTUATOR DESIGN}

The vertical displacement of the electro-thermal actuator is achieved by the thermal mismatch between the hot and the cold arms. After the flip-chip assembly and transfer, the MEMS vertical displacement should be controllable from $2 \mu \mathrm{m}$ to 0.5 or $0.2 \mu \mathrm{m}$. Such a displacement is achieved by the differential expansions of the arms resulting from different temperature distributions. Figure 3 illustrates the temperature distributions of a design with the cold arm placed in the middle between the two hot arms. The distribution is presented along each arm from the top intersection point to the bottom point anchored to the ceramics substrate. Clearly indicated, the cold arn is really not cold. In fact, its temperature can be higher than the corresponding hot arm when the length is longer than $150 \mu \mathrm{m}$. When the temperature differentials are non-linear, repeatable vertical displacements are difficult to achieve. The solution is to place the hot arm in the middle for a consistent temperature differential for repeatable upand-down plate movements. The improved temperature distributions are shown in Figure 4.

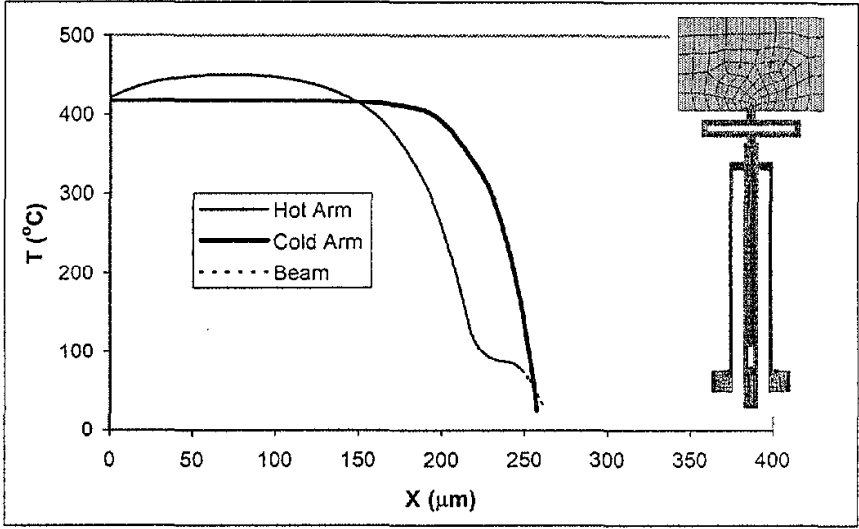

Figure 3: Temperature distributions of an actuator with the cold arm in the middle

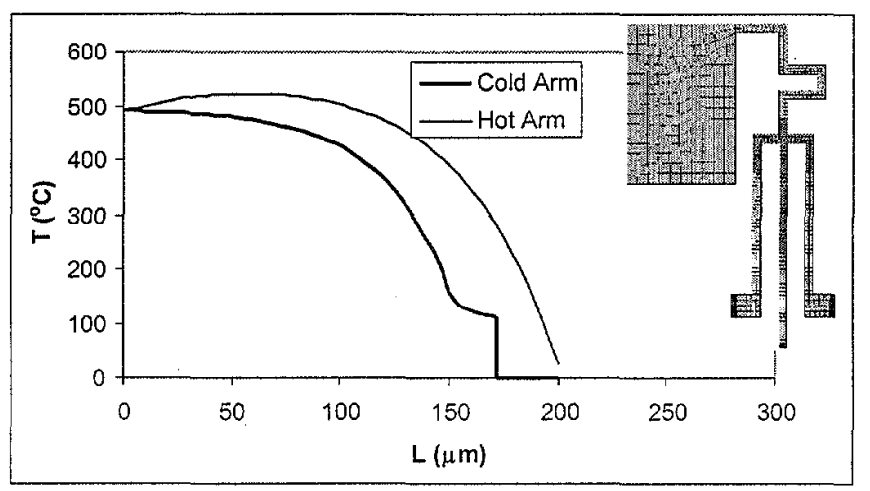

Figure 4: Temperature distributions of an improved actuator with the hot arm in the middle

\section{RF DESIGN AND CHARACTERIZATION}

There were two objectives for the RF design: controlling the selfresonant frequency and de-coupling the actuators from the capacitor plate for low RF losses. In addition to capacitance, there is always spurious inductance associated with the plate, the actuator and the connections between the two. With a poor inductance design, the MEMS could reach the undesirable selfresonance around $30 \mathrm{GHz}$ (see Figure 5)[6]. The design was improved to solve this resonance problem. As shown in Figure 6, for low inductance and low loss, a shunt-mounted configuration replaced the series-mounted configuration reported in [6].

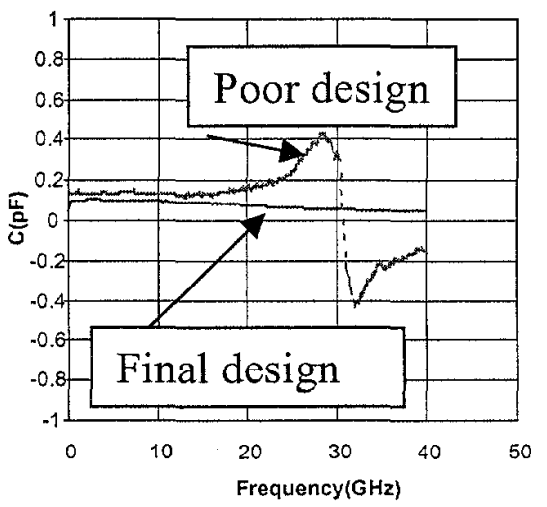

Figure 5: Measured capacitance up to $40 \mathrm{GHIz}$ 


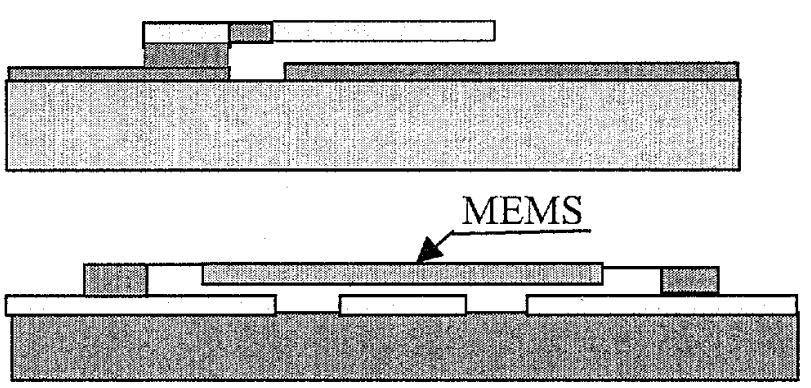

Figure 6: Series-mounted configuration (top) with left-to-right RF transmission and shunt-mounted (bottom) configuration with outof-plane RF transmission

With the shunt-mounted configuration, the inductance of the actuators was further designed through simulations. Figure 7 illustrates the configuration simulated, and Figure 8 presents the resonant frequencies corresponding to different actuator widths. Wider actuators resulted in low inductance that increased the resonant frequency. In addition to the width, the length and the number of actuators could be changed to control the resonant frequencies.

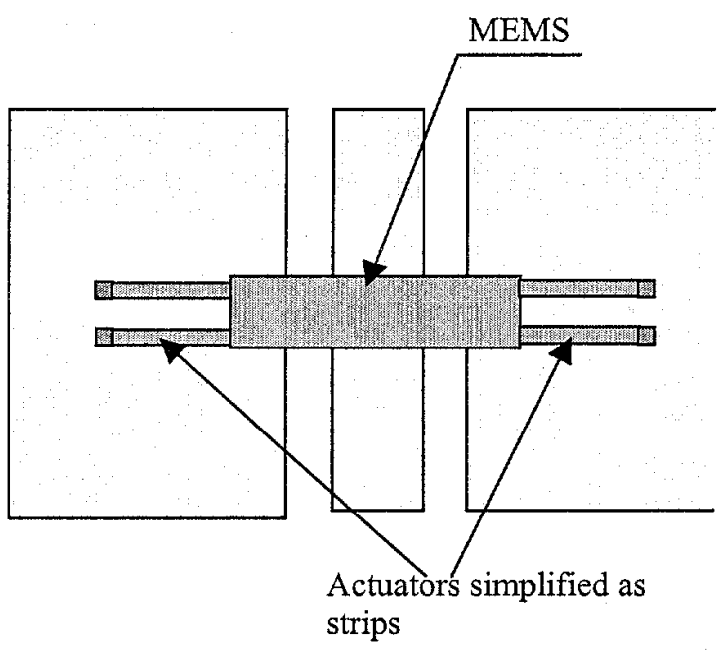

Figure 7: MEMS with actuators simulated

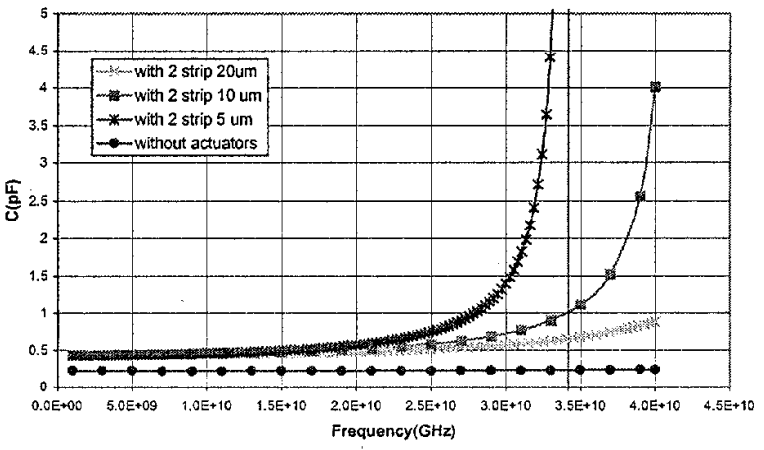

Figure 8: Effects of actuators on resonant frequencies

With the new configuration and enhanced MEMS design, the resonant frequency was increased from $30 \mathrm{GHz}$ to a much higher value. As shown in Figure 5, there is no resonance up to $40 \mathrm{GHz}$. The new MEMS variable capacitor could be designed with low inductance for millimeter-wave applications.

However, low inductance was not always preferred. As shown in Figure 9, the actuators should be isolated from the MEMS plate in order to reduce the RF losses. As shown in Figure 10, the decoupling would become effective when the actuator inductance (La) is high. With high La, the RF current would be limited to the MEMS plate region and could avoid unnecessary losses in actuators. For good design, we want $\mathrm{L} a$ to be large but $\mathrm{L}_{1}$ to be small.

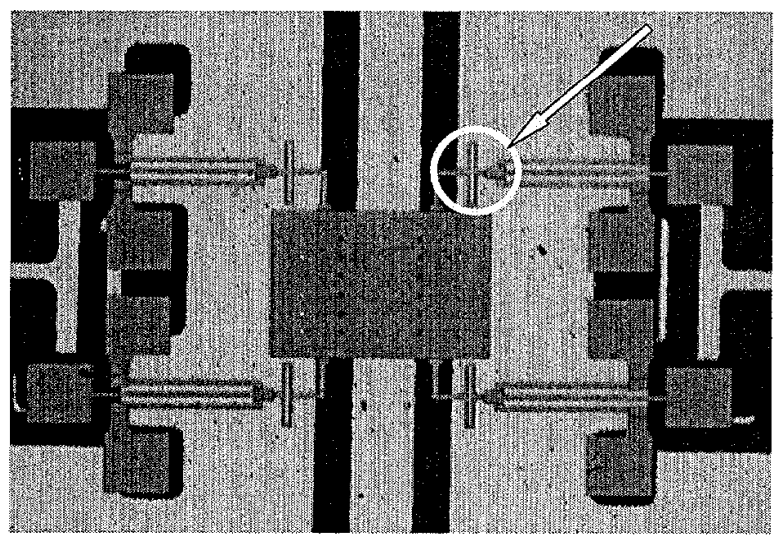

Figure 9: Coupling between the capacitor plate and the actuator

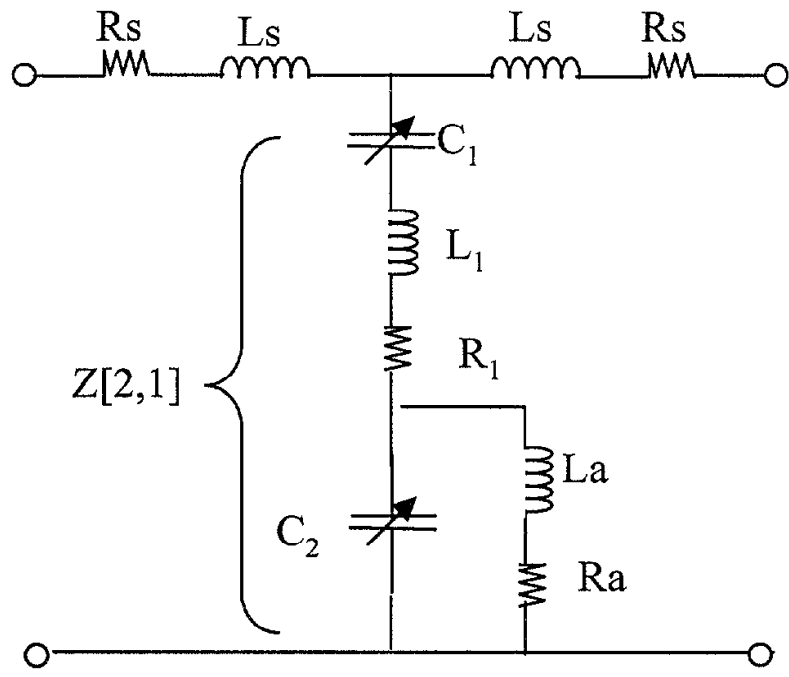

Figure 10: Equivalent circuits for the shunt-mounted MEMS variable capacitor

The S-parameters of the MEMS variable capacitor were measured using HP 8510B Network Analyzer. Figure 11 shows the Sparameters at different capacitance values controlled by the voltages applied to the thermal actuators. The capacitance change achieved a ratio up to $2.7: 1$ for the case measured. The ratio was directly related to the displacement of the thermal actuator, and could be improved further. Air's dielectric loss is negligible; therefore, the MEMS-based capacitor achieved a very impressive quality factor (Q) that was about 300 at $0.1 \mathrm{pF}$ and $10 \mathrm{GHz}$. Figure 12 presents the $S$-parameters measured. These parameters were used to derive the Z-parameters, which were used to establish the equivalent lump elements in the T-circuit as shown in Figure 10. 
The equivalent resistance was the $Z[2,1]+\mathrm{Rs} / 2$. From the resistance, the quality factor $(Q)$ could be estimated.

Figure 13 shows the equivalent resistances in the frequency range up to $40 \mathrm{GHz}$. Three interesting observations are listed below.

1. The resistance dropped substantially when the frequency was above $10 \mathrm{GHz}$. Above that frequency, the actuators were decoupled from the MEMS plate and the RF losses were reduced.

2. The equivalent resistance's error bars were close to the mean values. Thus, the resistances were so low that they were in the "noise" region. It is very difficult to measure these resistance values precisely. But, clearly indicated, the resistances, i.e. RF losses, were very very low even at $40 \mathrm{GHz}$. It should be noted that the gold thickness of the MEMS plate was only $0.5 \mu \mathrm{m}$ that is the standard thickness offered by the MUMPS. With a thicker gold layer, the RF losses could be reduced further.

3. The quality factor could be estimated using $Q=1 /(\omega R C)$. With $\mathrm{R}=0.5 \Omega$, the $\mathrm{Q}$ was about 300 at $0.1 \mathrm{pF}$ and $10 \mathrm{GHz}$.

The achieved quality factor of 300 at $0.1 \mathrm{pF}$ and $10 \mathrm{GHz}$ is very impressive. The MEMS variable capacitor demonstrated has the outstanding $\mathrm{RF}$ performance even in the millimeter-wave range.

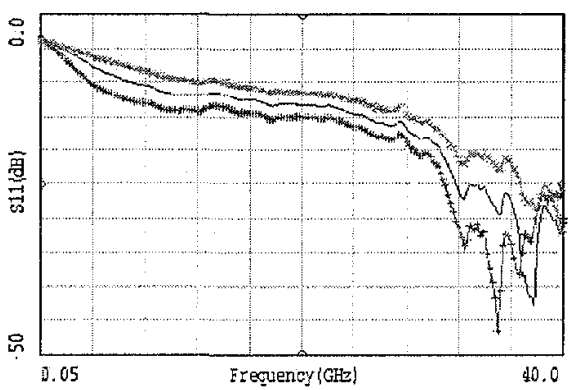

(a)

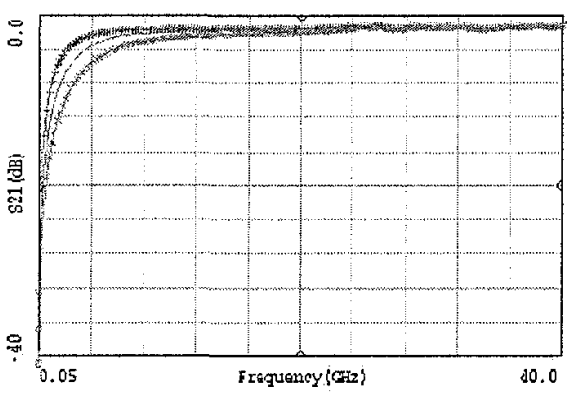

(b)

Figure 11: S-parameters at different capacitance values controlled by different biased voltages

\section{SUMMARY}

We have demonstrated a MEMS variable capacitor with outstanding RF performance up to millimeter-wave range. More importantiy, these MEMS were fabricated using standard siliconMEMS foundry service, MUMPS. The excellent performance of our devices was achieved by using a novel flip-chip assembly with silicon removal technology and by appropriate actuator and RF designs.

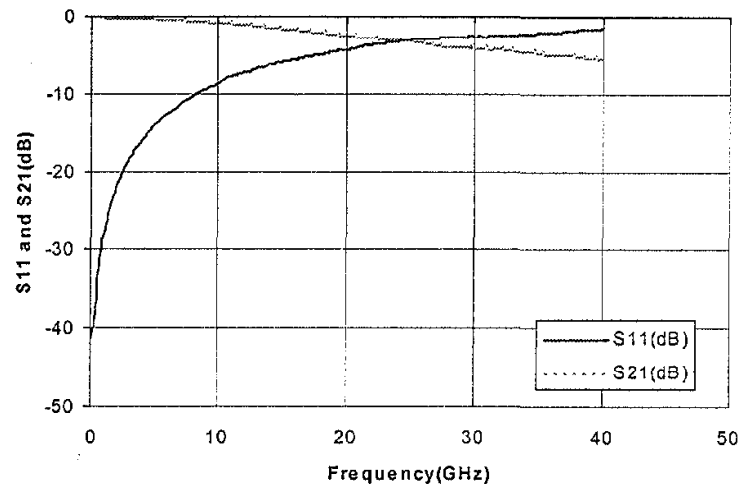

Figure 12: S-parameters for the MFMS variable capacitor

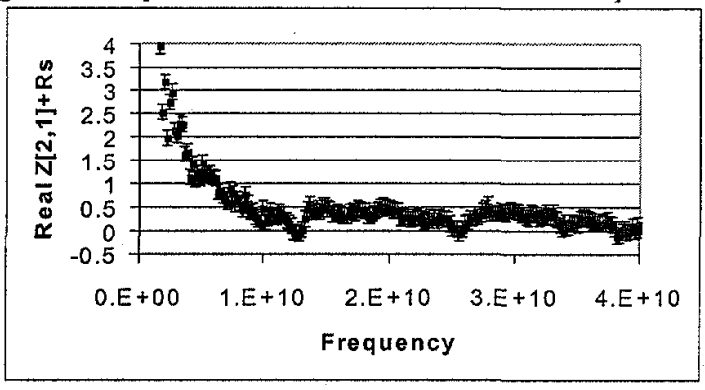

Figure 13: Measured resistance values up to $40 \mathrm{GHz}$

\section{ACKNOWLEDGMENTS}

Research was supported by DARPA/DSO's FAME (frequency agile materials for electronics) with the grant number F33615-98C-5429. The RF measurements were conducted at the National Institute of Standards and Technologies at Boulder, CO.

\section{REFERENCES}

1. L. Larson, et al., "Micromachined microwave actuator (MIMAC) technology - a new tuning approach for microwave integrated circuits," IEEE Microwave and Millimeter-wave Monolithic Circuits Symposium, 1991, pp. 27-30.

2. A. Dec and K. Suynama, "Micromachined varactor with wide tuning range," Electronics Letters, Vol. 33, No. 11, May 1997, pp. 922-924.

3. A. Dec and K. Suynama, "A $2.4 \mathrm{GHz}$ CMOS LC VCO using micromachined variable capacitors for frequency tuning," 1999 IEEE MTT-S International Microwave Symposium Digest, pp. 79-82,

4. D. Young and B. Boser, "A micromachined variable capacitor for monolithic low-noise VCOs," Solid-state Sensor and Actuator Workshop, June 2-6, 1996. pp. 86-89.

5. K.F. Harsh, et al., "Flip-chip assembly for Si-based RF MEMS," Technical Digest of $12^{\text {th }}$ IEEE International Conference on MicroElectroMechanical Systems - MEMS '99, pp. 273-278, 17-21 January 1999, Orlando, FL.

6. Z. Feng, et al., "Design and modeling of RF MEMS tunable capacitors using electro-thermal actuators," 1999 IEEE MTTS International Microwave Symposium Digest, pp. 15071510. 